\title{
Nano-copper-bearing stainless steel promotes fracture healing by accelerating the callus evolution process
}

This article was published in the following Dove Press journal:

International Journal of Nanomedicine

Number of times this article has been viewed

\author{
Lei Wang, ${ }^{1, *}$ Guoyuan Li, ${ }^{1}, *$ \\ Ling Ren, ${ }^{2, *}$ Xiangdong \\ Kong,' Yugang Wang,' \\ Xiuguo Han, ' Wenbo Jiang, ${ }^{3}$ \\ Kerong Dai,' Ke Yang, ${ }^{2}$ \\ Yongqiang $\mathrm{Hao}^{\prime}$ \\ 'Shanghai Key Laboratory of \\ Orthopaedic Implants, Department of \\ Orthopaedics, Shanghai Ninth People's \\ Hospital, Shanghai JiaoTong University \\ School of Medicine, Shanghai, \\ ${ }^{2}$ Special Materials and Device \\ Research Department, Institute of \\ Metal Research, Chinese Academy \\ of Sciences, Shenyang, ${ }^{3}$ Medical 3D \\ Printing Innovation Research Center, \\ Shanghai Ninth People's Hospital, \\ Shanghai JiaoTong University School \\ of Medicine, Shanghai, People's \\ Republic of China \\ *These authors contributed equally \\ to this work
}

Correspondence: Yongqiang Hao Shanghai Key Laboratory of Orthopaedic Implants, Department of Orthopaedics, Shanghai Ninth People's Hospital, Shanghai JiaoTong University School of Medicine, 639 Zhizaoju Road, Shanghai 20001 I, People's Republic of China Tel +86 2I 2327 I699

Fax +86 21 63136856

Email hyq_9hospital@hotmail.com

Ke Yang

Institute of Metal Research, Chinese Academy of Sciences, 72 Wenhua Road, Shenyang II0016, People's Republic of China

Tel/fax +86242397 I880

Email kyang@imr.ac.cn
Abstract: Treatment for fractures requires internal fixation devices, which are mainly produced from stainless steel or titanium alloy without biological functions. Therefore, we developed a novel nano-copper-bearing stainless steel with nano-sized copper-precipitation (317L-Cu SS). Based on previous studies, this work explores the effect of $317 \mathrm{~L}-\mathrm{Cu}$ SS on fracture healing; that is, proliferation, osteogenic differentiation, osteogenesis-related gene expression, and lysyl oxidase activity of human bone mesenchymal stem cells were detected in vitro. Sprague-Dawley rats were used to build an animal fracture model, and fracture healing and callus evolution were investigated by radiology (X-ray and micro-CT), histology (H\&E, Masson, and safranin $\mathrm{O}$ /fast green staining), and histomorphometry. Further, the $\mathrm{Cu}^{2+}$ content and Runx2 level in the callus were determined, and local mechanical test of the fracture was performed to assess the healing quality. Our results revealed that $317 \mathrm{~L}-\mathrm{Cu}$ SS did not affect the proliferation of human bone mesenchymal stem cells, but promoted osteogenic differentiation and the expression of osteogenesis-related genes. In addition, 317L-Cu SS upregulated the lysyl oxidase activity. The $\mathrm{X}$-ray and micro-CT results showed that the callus evolution efficiency and fracture healing speed were superior for 317L-Cu SS. Histological staining displayed large amounts of fibrous tissues at 3 weeks, and cartilage and new bone at 6 weeks. Further, histomorphometric analysis indicated that the callus possessed higher osteogenic efficiency at 6 weeks, and a high $\mathrm{Cu}^{2+}$ content and increased Runx2 expression were observed in the callus for 317L-Cu SS. Besides, the mechanical strength of the fracture site was much better than that of the control group. Overall, we conclude that $317 \mathrm{~L}-\mathrm{Cu}$ SS possesses the ability to increase $\mathrm{Cu}^{2+}$ content and promote osteogenesis in the callus, which could accelerate the callus evolution process and bone formation to provide faster and better fracture healing.

Keywords: nano-sized copper, lysyl oxidase, osteogenesis, fracture healing, callus evolution

\section{Introduction}

As access to motorized transportation, natural calamities and industrial accidents have increased throughout the developing world, there has been a dramatic increase in trauma and bone fractures. ${ }^{1,2}$ Bone fracture is one of the most common injuries, which is associated with high treatment costs exceeding billions of dollars, societal productivity loss, and individual disability. ${ }^{3,4}$ With the development of orthopedic surgical techniques and implant materials, the prognosis of fracture is markedly improved. Fracture healing is an intricate coordination of various cellular and mechanosensitive processes; however, $\sim 5 \%-10 \%$ of fractured bones end in nonunion and/or incomplete healing. ${ }^{5}$ Understanding the biomechanical aspects of the healing process in detail or designing new implant materials with better biological activity may be an effective 
way to properly create the optimal healing environment for an injured bone. ${ }^{6}$

The principal treatment for fractures involves early treatment, anatomic reduction, and rigid fixation. Open fractures, closed fractures with failed manual reduction, and obsolete fractures without ideal function are treated with operation. Generally, surgical treatments for these fractures need internal fixation devices such as screws, intramedullary nails, and internal plates, which are mainly composed of stainless steel or titanium alloy. Although these materials meet the requirements of clinical application in terms of biocompatibility and mechanical properties, the incidence of fracture-related complications such as infection, delayed union, and nonunion remain a major clinical problem that further increases the suffering and financial burden of fracture patients..$^{7-10}$ Therefore, imparting antibacterial and/ or osteogenic properties to the existing metal implants is crucial for improving the prognosis of fracture and reducing postoperative complications.

In recent years, many elements or particles, such as Ag, $\mathrm{Mg}, \mathrm{ZnO}$ and $\mathrm{TiO}_{2}$, have been proven to possess excellent antibacterial and/or osteogenic properties. ${ }^{11-13}$ But these elements or particles are difficult to directly incorporate into the stainless steel. Therefore, we set our sights on a commonly used metallurgy element - copper $(\mathrm{Cu}) . \mathrm{Cu}$ is one of the indispensable metal elements in the human body that participates in the regulation of a variety of key enzymes such as lysyl oxidase (LOX), ${ }^{14}$ superoxide dismutase, tyrosinase, ${ }^{15}$ and dopamine- $\beta$-hydroxylase. ${ }^{16}$ It also plays important roles in multiple systems, including the nervous system, cardiovascular system, and immune system. ${ }^{17}$ Also, recent intensive research has revealed that $\mathrm{Cu}$ provides good antibacterial, ${ }^{18-20}$ osteogenic, ${ }^{20}$ and antineoplastic properties, ${ }^{21}$ which is potentially advantageous for the treatment of orthopedic diseases. Unfortunately, $\mathrm{Cu}$ is not directly used as an implant material in clinical applications, except for in female intrauterine devices, ${ }^{22}$ due to its inflammatory property and cytotoxicity. Although some researchers deposited $\mathrm{Cu}$ coatings on the surfaces of orthopedic implants through different processing techniques, ${ }^{23,24}$ the $\mathrm{Cu}$ layer might lead to massive release of $\mathrm{Cu}$ ions or tiny $\mathrm{Cu}$ particles, which may cause a relatively severe and persistent foreign body reaction (FBR) in vivo. ${ }^{25}$ Therefore, to overcome this limitation, our team directly incorporated $\mathrm{Cu}$ nanoparticles in the medical grade $317 \mathrm{~L}$ stainless steel (317L SS) substrate to obtain a novel stainless steel with nano-sized $\mathrm{Cu}$-precipitation, with a nominal chemical composition of Fe-19Cr-14Ni-3Mo-4.5Cu in $\mathrm{wt}^{\%} \%{ }^{26}$ The major advantage of $\mathrm{Cu}-\mathrm{SS}$ is that the $\mathrm{Cu}$ nanoparticle precipitates are evenly distributed in the stainless steel substrate from which a trace small amount of $\mathrm{Cu}^{2+}$ can continuously release $\left(0.137 \mathrm{ng} / \mathrm{cm}^{2} /\right.$ day $)$ to further reduce the degree of FBR caused by $\mathrm{Cu}^{2+}$ and contribute to the biological functions of the new material. ${ }^{24}$ As expected, our initial studies confirmed that the new material possessed good biocompatibility ${ }^{27}$ as well as superior antibacterial and osteogenic properties. ${ }^{26,28}$ To determine the clinical value of $\mathrm{Cu}-\mathrm{SS}$ in orthopedics, the present research will focus on the basic effect of $\mathrm{Cu}-\mathrm{SS}$ on fracture healing and remodeling, and elucidate the related mechanism, finally providing a theoretical basis for its clinical transformation.

\section{Materials and methods}

\section{Implant material fabrication}

A 317L-Cu stainless steel (317L-Cu SS) was designed and fabricated by the Institute of Metal Research, Chinese Academy of Sciences, Shenyang, China. The nominal chemical compositions and the detailed fabrication process have been described in our previous study. ${ }^{26}$ Samples were cut into round disks in 2 sizes: large disks with $10 \mathrm{~mm}$ diameter and $1 \mathrm{~mm}$ thickness and small disks with $5 \mathrm{~mm}$ diameter and $1 \mathrm{~mm}$ thickness for use in in vitro experiments. Intramedullary nails, which are used for femur fracture fixation in Sprague-Dawley (SD) rats in vivo, were processed by colddrawing $317 \mathrm{~L}-\mathrm{Cu}$ SS to $1.5 \mathrm{~mm}$ diameter, and were cut into suitable length for each rat femur. A medical grade 317L SS with the same sample shape and dimension was used as the control material. These samples were mechanically polished with silicon carbide papers down to 2000 grade and were ultrasonically washed in acetone and ethanol. All the samples were sterilized before performing the experiments.

\section{Mechanical properties}

Tensile tests were carried out at ambient temperature using an Instron-8872 universal testing machine (Instron Corporation, Boston, MA, USA) at a constant stretching rate of $0.5 \mathrm{~mm} / \mathrm{min}$ with standard specimens of M10× $\Phi 5 \mathrm{~mm}$. Five parallel samples were prepared for each test.

\section{Scanning electron microscope (SEM) detection}

Samples for SEM observation were firstly carefully washed. Subsequently, the samples were dried with graded alcohol. The surface microstructure of the samples was observed using a SEM (Joel JSM-6310LV; JEOL Ltd., Tokyo, Japan).

\section{Cell culture}

Human bone mesenchymal stem cells (hBMSCs) were isolated and expanded via the modification of standard methods, as previously described. ${ }^{29}$ All studies were carried 
out in accordance with the policies of, and with approval from, the Ninth People's Hospital of Shanghai Jiao Tong University. The donor (male, 41 year) was healthy without metabolic diseases, inherited illnesses, or other diseases that may affect the current study. Briefly, bone marrow from the donor was taken after written informed consent using guidelines approved by the Ethical Committee on the Use of Human Subjects at the Shanghai Jiao Tong University. Cells were grown in $\alpha$-MEM (Gibco, Grand Island, NY, USA) supplemented with 10\% fetal bovine serum FBS (Gibco) containing $100 \mathrm{U} / \mathrm{mL}$ penicillin, and $100 \mu \mathrm{g} / \mathrm{mL}$ streptomycin (Hyclone, Logan, UT, USA) in a humidified atmosphere at $37^{\circ} \mathrm{C}$ with $5 \% \mathrm{CO}_{2}$. The media were changed every 3 days until cells were confluent.

\section{Cell viability test}

Cell Counting Kit-8 (CCK-8) assay was performed according to the manufacturer's instructions (Dojindo, Kumamoto, Japan) to evaluate the viability of hBMSCs under the influence of $317 \mathrm{~L}-\mathrm{Cu}$ SS. First, the small disks were placed in the 96 -well plates and $3 \times 10^{3} \mathrm{hBMSC}$ were seeded on each disk. The cells were cultured in the aforementioned medium and conditions for 1, 3, 5, and 7 days. The culture medium was removed before the CCK-8 examination. Subsequently, $100 \mu \mathrm{L}$ of $\alpha$-MEM medium and $10 \mu \mathrm{L}$ of CCK-8 solution were added to each sample, followed by incubation at $37^{\circ} \mathrm{C}$ for $2.5 \mathrm{~h}$. The absorbance of CCK-8, which represents cell viability, was recorded at $450 \mathrm{~nm}$ using a microplate reader (BioTek, Winooski, VT, USA).

\section{Osteogenic differentiation of hBMSCs}

The effect of $317 \mathrm{~L}-\mathrm{Cu}$ SS on the osteogenic differentiation of hBMSCs was evaluated from 2 aspects: release of $\mathrm{Cu}^{2+}$ and the interface. To detect the effect of $\mathrm{Cu}^{2+}$ released from $317 \mathrm{~L}-\mathrm{Cu}$ SS on the osteogenic differentiation of hBMSCs, a 24-well Transwell chamber (Merck Millipore, Billerica, MA, USA) was used to build a special system in which $2 \times 10^{4} \mathrm{hBMSC}$ sere seeded in the 24 -well plate and a small disk was placed in the upper chamber. After $24 \mathrm{~h}$, the culture medium was replaced with osteogenic induction medium containing $\alpha$-MEM supplemented with $10 \% \mathrm{FBS}, 0.1 \mu \mathrm{M}$ dexamethasone (Sigma, St Louis, MO, USA), $50 \mu \mathrm{M}$ ascorbate acid (Sigma), and $10 \mathrm{mM} \beta$-glycerophosphate sodium (Sigma) to promote the osteogenic differentiation of hBMSCs. After 4 days, alkaline phosphatase (ALP) staining and activity determination were carried out. On the other hand, in order to determine the effect of the material interface on the osteogenic differentiation of hBMSCs, 6 groups were formed: 1) hBMSCs + 317L-Cu SS + osteogenic induction medium; 2) hBMSCs + 317L SS + osteogenic induction medium; 3) hBMSCs + 317L-Cu SS; 4) hBMSCs + 317L SS; 5) 317L-Cu SS; and 6) 317L SS. The same amount of hBMSCs $\left(2 \times 10^{4}\right)$ was directly seeded on the surface of the large disks and the culture medium was changed to osteogenic induction medium after 3 days. ALP staining and alizarin red staining were performed after 7 and 21 days, respectively. The osteogenic induction medium was renewed every 3 days throughout the study period.

\section{ALP staining and activity}

ALP staining was performed according to the procedure provided by the manufacturer (Jiancheng, Nanjing, China) at the aforementioned time points. In short, hBMSCs were fixed using buffered formalin for $30 \mathrm{~s}$, after which the cells were washed with distilled water twice and then stained with a staining reagent for $30 \mathrm{~min}$. The cells were then photographed or scanned. The level of ALP activity was determined at the aforementioned time points using p-nitrophenyl phosphate (pNPP) (Sigma), as previously described. ${ }^{30}$ First, the samples were washed three times with PBS and then lysed in $0.2 \%$ Triton X-100 solution using 4 freeze-thaw cycles. The cell lysate $(50 \mu \mathrm{L})$ was mixed with $50 \mu \mathrm{L}$ pNPP $(1 \mathrm{mg} / \mathrm{mL})$ in $1 \mathrm{M}$ diethanolamine buffer containing $0.5 \mathrm{mM} \mathrm{MgCl}_{2}(\mathrm{pH}$ 9.8) and incubated at $37^{\circ} \mathrm{C}$ for $45 \mathrm{~min}$ on a platform shaker. The reaction was terminated by adding $25 \mu \mathrm{L}$ of $3 \mathrm{~N} \mathrm{NaOH}$ per $100 \mu \mathrm{L}$ of reaction mixture. ALP was quantified by measuring the absorbance at $405 \mathrm{~nm}$ (BioTek), and the total protein content was determined by measuring the absorbance at $562 \mathrm{~nm}$ using a Pierce Protein Assay Kit (Thermo Fisher Scientific, Waltham, MA, USA) with bovine serum albumin as the standard. The ALP activity was normalized to the total protein content for each time point.

\section{Alizarin red staining}

After 3 weeks of osteogenic induction for hBMSCs seeded on the surface of the materials, alizarin red staining was carried out. Briefly, the cells were fixed in 10\% formalin and incubated with $1 \%$ alizarin red S (Sigma) solution for $10 \mathrm{~min}$ at room temperature. The stained cells were then photographed. Thereafter, mineralized nodules were dissolved with $10 \%$ cetylpyridinium chloride (Sigma) for semi-quantitative analysis by examining the absorbance at $562 \mathrm{~nm}$.

\section{Real-time polymerase chain reaction (PCR)}

Large disks were used in this test. First, the disks were placed at the bottom of 24 -well plates and $\sim 2 \times 10^{4}$ cells were seeded on the surface of each large disk, followed by incubation 
at $37^{\circ} \mathrm{C}$ in $5 \% \mathrm{CO}_{2}$ and saturated humidity for 7 days. Thereafter, total RNA was isolated using the AxyPrep ${ }^{\mathrm{TM}}$ Multisource Total RNA Miniprep Kit (Axygen, Corning, NY, USA), and an equivalent amount of RNA was converted into complementary DNA (complementary deoxyribonucleic acid [cDNA]) with PrimeScript ${ }^{\mathrm{TM}} \mathrm{RT}$ reagent Kit (Takara, Kusatsu, Japan). Subsequently, real-time PCR was performed using an ABI 7500 Sequencing Detection System and SYBR ${ }^{\circledR}$ Premix Ex Taq (Takara). All the procedures were performed according to the manufacturer's protocols. The employed cycling condition was 40 cycles at $95^{\circ} \mathrm{C}$ for $5 \mathrm{~s}$ and $60^{\circ} \mathrm{C}$ for $34 \mathrm{~s}$. The primer sequences are shown in Table 1 . The comparative $2-\Delta \Delta \mathrm{CT}$ method was used to calculate the relative expression level of each target gene with glyceraldehyde-3-phosphate dehydrogenase (GAPDH) as the housekeeping gene.

\section{Enzyme-linked immunosorbent assay (ELISA)}

When hBMSCs were affected by $317 \mathrm{~L}-\mathrm{Cu}$ SS and $317 \mathrm{~L}$ $\mathrm{SS}$, the amounts of LOX secreted into the medium were determined by ELISA (Xinle Bio, Shanghai, China). First, $10^{4}$ cells were seeded onto each disk, and the culture medium was collected and centrifuged at 10,000 rpm for $5 \mathrm{~min}$ to clear the remaining impurities and particles. The test was performed according to the manufacturer's instructions and was repeated three times. Finally, the mean values of triplicate samples were used to calculate the concentrations of LOX based on a standard curve obtained with the standard LOX provided in the kit.

\section{LOX activity assay}

The culture media of 317L-Cu SS group and 317L SS group collected through the aforementioned method were added

Table I Primer sequences

\begin{tabular}{lll}
\hline Gene & \multicolumn{2}{l}{ Primer sequences $\left(\mathbf{5}^{\prime} \mathbf{-} \mathbf{3}^{\prime} \mathbf{)}\right.$} \\
\hline Human & Forward & CCT GCA CCA CCA ACT GCT TA \\
GAPDH & Reverse & AGG CCA TGC CAG TGA GCT T \\
Human & Forward & CGG AAT GCC TCT GCT GTT AT \\
Runx2 & Reverse & TGT GAA GAC GGT TAT GGT CAA G \\
Human & Forward & CCA CGC CCT TTG CTT TAT CT \\
ALP & Reverse & ATG GTC TGA GCC TCC TCT TT \\
Human & Forward & CAT ATG ATG GCC GAG GTG ATA G \\
OPN & Reverse & AGG TGA TGT CCT CGT CTG TA \\
Human & Forward & CCT GTC TGC TTC CTG TAA ACT C \\
Collagen I & Reverse & GTT CAG TTT GGG TTG CTT GTC \\
Human & Forward & CAG GAT GGG CAG AGG TAT AAT G \\
Collagen II & Reverse & GAG GCA GTCT TTC ACG TCT T \\
\hline
\end{tabular}

Abbreviations: $A L P$, alkaline phosphatase; GAPDH, glyceraldehyde-3-phosphate dehydrogenase; OPN, osteopontin. into a 96-well plate with $50 \mu \mathrm{L} /$ well for the detection of LOX activity. The subsequent experimental steps followed the manufacturer's instruction for the LOX Activity Assay Kit (Abcam, Cambridge, MA, USA). In short, $50 \mu \mathrm{L}$ of premade reaction solution was dropped into each well containing the culture medium. The mixed solution was then cultured in a cell incubator in the dark for $20 \mathrm{~min}$. Finally, the fluorescence intensity at $\mathrm{Ex} / \mathrm{Em}=530 / 590 \mathrm{~nm}$, which indirectly reflects the LOX activity, was detected using a microplate reader (BioTek).

\section{Experimental design and surgical procedure}

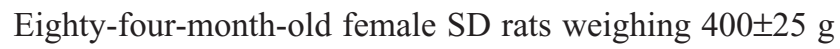
(Shanghai Sipper BK Laboratory Animals Ltd, Shanghai, China) were used for this study. They were maintained in plastic cages in a room with $12 \mathrm{~h}$ day/night cycle and ambient temperature of $21^{\circ} \mathrm{C}$, and were allowed ad libitum access to water and standard laboratory pellets. All the animal related studies in this research were performed in compliance with the Chinese laws on animal experimentation and were approved by the Ethical Committee of Shanghai Jiao Tong University.

The rats were randomly divided into $317 \mathrm{~L}-\mathrm{Cu}$ SS group and $317 \mathrm{~L}$ SS group. During surgery, they were first anesthetized with $1 \%$ sodium pentobarbital $(40 \mathrm{mg} / \mathrm{kg})$ by intraperitoneal injection on a clean bench. The right leg of each rat was shaved and disinfected with alcohol. A skin incision was made followed by blunt dissection of the subcutaneous tissue and muscles to expose the femur. At the mid-thigh diaphyseal location, a transverse osteotomy was performed immediately with a reciprocating saw and was stabilized using the intramedullary nails made of $317 \mathrm{~L}-\mathrm{Cu}$ SS and $317 \mathrm{~L} \mathrm{SS}$. This was followed by suturing the muscle and skin to successfully accomplish the establishment of an animal fracture model. Tetracycline (50 mg/kg, Sigma) and calcein (20 mg/kg, Sigma) were subcutaneously injected at 14 and 7 days, respectively, before sacrifice.

\section{X-ray analysis}

At 3, 6, and 9 weeks post-surgery, animals from the 2 groups were randomly executed by cervical dislocation, and the right femurs were carefully isolated. Afterward, conventional anteroposterior and lateral-view plain radiographs of the rat femurs were performed using an X-ray imaging system for small animal studies (12 s, $26 \mathrm{kV}$; Faxitron MX20, Lincolnshire, IL, USA) to evaluate the progress in bone healing and periosteal callus formation. 


\section{Micro CT}

After the intramedullary nails were carefully removed, the rat femurs were wrapped in wet gauze and fitted in a suitable tube for micro-CT scanning ( $\mu \mathrm{CT}$ 80; SCANCO Medical AG, Bassersdorf, Switzerland). In brief, the scan range was set as $9.6 \mathrm{~mm}$ (600 slides), and the fracture line was set in the middle of this range. The resolution was $16 \mu \mathrm{m} /$ voxel and $1,024 \times 1,024$ pixels. The region of interest was selected from the 2D images with a standardized threshold $(>165)$ of the mineralized tissue. Three-dimensional (3D) reconstruction of the external callus was performed and the following histomorphometric parameters were included in the analysis: bone mineral density (BMD), bone volume/tissue volume (BV/TV), trabecular number (Tb. N), trabecular thickness (Tb. Th), and trabecular spacing (Tb. Sp).

\section{Decalcification of femurs}

At 3, 6, and 9 weeks post-surgery, some of the right femurs from each group were first fixed in $4 \%$ paraformaldehyde in PBS overnight at $4{ }^{\circ} \mathrm{C}$, and then immediately decalcified in $10 \%$ EDTA for $\sim 8$ weeks at room temperature in a rotary mixing instrument. The callus area was retained, dehydrated in an ethanol series $(75 \%, 80 \%, 85 \%, 90 \%, 95 \%$, and $100 \%)$, and then embedded in paraffin. Subsequently, the tissues were serially cut into $5 \mu \mathrm{m}$ thick sections, which were then deparaffinized and subjected to H\&E, Masson, and safranin $\mathrm{O}$ staining.

\section{H\&E staining}

Some of the sections obtained previously were first preheated in an air oven at $60^{\circ} \mathrm{C}$, followed by deparaffinization and rehydration in xylene and ethanol solutions (reducing concentration $95 \%-70 \%)$. The sections were then successively soaked in $\mathrm{H} \& \mathrm{E}$ dyes for $\mathrm{H} \& \mathrm{E}$ staining. After dehydration with graded alcohol and safranin $\mathrm{O}$ staining with phenolic xylene and xylene, histological observation was performed using an optical microscope (Leica DM 4000B; Leica Microsystems, Wetzlar, Germany). Subsequently, the fracture healing process was evaluated.

\section{Safranin O/fast green staining of cartilage}

The dewaxed sections were successively rinsed in alcohol and PBS and soaked in Weigert's hematoxylin for $3 \mathrm{~min}$, followed by staining in $0.02 \%$ aqueous fast green (Sigma) for another $3 \mathrm{~min}$. The sections were briefly washed in $1 \%$ acetic acid and spin-dry the remaining fluid from the section. Subsequently, the sections were stained in $0.1 \%$ aqueous safranin O (Sigma) for a maximum of $3 \mathrm{~min}$. After dehydrating, clearing, and mounting, digital images were captured on an optical microscope (Leica DM 4000B; Leica Microsystems) to observe the newly formed bone and callus composition.

\section{Masson staining}

The sections were routinely deparaffinized and rehydrated in accordance with the aforementioned procedure. Weigert's hematoxylin was used to dye the cell nucleus for $5 \mathrm{~min}$. After rinsing with distilled water three times, the sections were stained in Masson-Ponceau-acid fuchsin solution for $10 \mathrm{~min}$. They were rinsed in $2 \%$ glacial acetic acid and then differentiated in phosphomolybdic acid for $4 \mathrm{~min}$. The sections were directly stained with aniline blue dye solution (Sigma). After dehydrating with ethanol series, clearing with xylene, and mounting with neutral resins, digital images were captured using an optical microscope (Leica DM 4000B) to analyze fiber formation in the early callus.

\section{Undecalcified bone histomorphometry}

After fixation, dehydration and clearing, the femurs used in this experiment were directly infiltrated and embedded in methyl methacrylate (MMA) without decalcification until the MMA solidified. These specimens were cut crosswise into $200 \mu \mathrm{m}$ thick slices perpendicular to the axis of the medullary cavity using a diamond saw (Leica SP1600; Leica Instruments, Nussloch, Germany) and were further ground and polished to $80 \mu \mathrm{m}$. Thereafter, the new bone formation rate was evaluated using a fluorescence microscope (Leica Q500 MC) without staining. The sections were subsequently stained with Van Gieson's picrofuchsin to observe new bone formation. Digital images were obtained using a microscope (Leica DM 4000B) with BioQuant OSTEO II software (BioQuant Image Analysis Corporation, Nashville, TN, USA), and the new bone area was semi-quantified by ImageJ.

\section{$\mathrm{Cu}^{2+}$ detection in callus and serum}

Callus $\mathrm{Cu}^{2+}$ detection was carried out by an inductively coupled plasma atomic emission spectrometer (IRIS Intrepid; Thermo Fisher Scientific, Waltham, MA, USA). In this measurement, callus tissues with the same weight were collected from each group, and then ground into homogenized tissue fluid for $\mathrm{Cu}^{2+}$ detection.

Serum $\mathrm{Cu}^{2+}$ detection has been explained in our previous article. ${ }^{27}$ In detail, blood was collected into procoagulant tubes by enucleation and then centrifuged at 4,000 rpm for 10 min after blood coagulation. Subsequently, the supernatant was transferred to new centrifuge tubes and the concentration 
of $\mathrm{Cu}^{2+}$ was determined according to the instructions of QuantiChrom ${ }^{\mathrm{TM}} \mathrm{Cu}$ Assay Kit (BioAssay Systems, Hayward, CA, USA).

\section{Western blot}

Cytoplasmic protein was prepared from the callus tissues using NE-PER ${ }^{\circledR}$ Nuclear and Cytoplasmic Extraction Reagents according to the manufacturer's instructions (Thermo Fisher Scientific). Ten percent sodium dodecyl sulfate polyacrylamide gel electrophoresis (SDS-PAGE) was used for protein (Runx2) electrophoresis. Different samples with an equal amount of protein were subjected to SDS-PAGE and then transferred to $0.22 \mu \mathrm{m}$ polyvinylidene fluoride (PVDF) membranes, which were then blocked with 5\% fat-free milk at room temperature for $1 \mathrm{~h}$ and subsequently incubated with anti-Runx 2 primary antibody (diluted to 1:1,000; Cell Signaling Technology, Danvers, MA, USA) overnight at $4^{\circ} \mathrm{C}$. Thereafter, the PVDF membranes were washed with Tris-buffered saline with Tween 20 and then incubated with a fluorescent secondary antibody, purchased from LI-COR (Lincoln, NE, USA), in the dark for $1 \mathrm{~h}$ at room temperature. Finally, the signals were detected using an Odyssey 2-color infrared fluorescence image system (LI-COR). Positive immunoreactive bands were quantified densitometrically and normalized by GAPDH.

\section{Biomechanical testing}

The biomechanical quality of bone during fracture healing was evaluated via the 3-point bending test using an AG-A20 KNA dynamic testing machine (Shimadzu, Nakagyo-ku Kyoto, Japan). Femurs from the 2 groups, with different time points, were randomly selected and placed between the 2 horizontal bars of the bending machine. The primary distance between the bars was set as $18 \mathrm{~mm}$ and the loading speed was $2 \mathrm{~mm} / \mathrm{min}$ until failure. Finally, the maximum compressive load was recorded.

\section{Statistical analysis}

Each sample was analyzed in triplicate, and the experiments were repeated 3 times. All the results are expressed as mean \pm standard error (SE) with n. Mean, SD, and $P$-values based on the 2-tailed $t$-test were calculated in Excel (Microsoft Corporation, Redmond, WA, USA) and $P<0.05$ was considered to be statistically significant.

\section{Results}

\section{Addition of $\mathrm{Cu}$ does not affect the mechanical properties of stainless steel}

The tensile properties, including yield strength $\left(R_{p 0.2}\right)$, tensile $\left(R_{m}\right)$ strength, elongation $(A)$, and reduction of area $(Z)$ of $317 \mathrm{~L}-\mathrm{Cu}$ SS meet the requirements of ISO 5832-1 standard for $317 \mathrm{~L} \mathrm{SS}$ as a surgical implant material. Moreover, the elastic modulus (E) of $317 \mathrm{~L}-\mathrm{Cu}$ SS is similar to that of $317 \mathrm{~L} \mathrm{SS}$ (Table 2). These results indicate that the addition of $\mathrm{Cu}$ does not affect the mechanical properties of stainless steel and thus it has the potential to be used as implant devices.

\section{I L L-Cu SS could significantly promote the osteogenic differentiation of hBMSCs without influence on their proliferation}

CCK-8 assay revealed that $317 \mathrm{~L}-\mathrm{Cu}$ SS had no significant impact on the proliferation of hBMSCs compared with $317 \mathrm{~L}$ SS for different time points (1, 3, 5, and 7 days) (Figure 1A). However, $317 \mathrm{~L}-\mathrm{Cu}$ SS could prominently promote the osteogenic differentiation of hBMSCs, possibly through $\mathrm{Cu}^{2+}$ regulation and interface stimulation. Our previous study has confirmed that $317 \mathrm{~L}-\mathrm{Cu}$ SS could continuously, slowly and steadily release $\mathrm{Cu}^{2+}$; therefore, in the special culture system mentioned previously, hBMSCs showed higher expression and activity of ALP after osteogenic induction for 4 days without directly contacting the interface of $317 \mathrm{~L}-\mathrm{Cu}$ SS (Figure 1B). Simultaneously, better osteogenic differentiation ability was observed when the hBMSCs were inoculated on the surface of $317 \mathrm{~L}-\mathrm{Cu}$ SS than on the surface of 317L SS. In detail, our results showed that $317 \mathrm{~L}-\mathrm{Cu}$ SS possessed the ability to slightly promote osteogenic differentiation in spite of no osteogenic induction. Further, it significantly promoted the expression and activity of ALP under osteogenic induction for 7 days (Figure 1C). After 21 days of osteogenic induction, alizarin red staining and quantitative evaluation showed that $317 \mathrm{~L}-\mathrm{Cu}$ SS enhanced the mineralization process in osteogenic differentiation (Figure 1D). Besides, the results of real-time PCR indicated that $317 \mathrm{~L}-\mathrm{Cu}$ SS could significantly upregulate the expression of Runx2, osteopontin and Collagen I. It could also promote the expression of $A L P$, but without significant statistical difference (Figure 1E). Collectively, these results suggest that $317 \mathrm{~L}-\mathrm{Cu} \mathrm{SS}$ is conducive to osteogenesis of hBMSCs, which might be related to the

Table 2 Tensile and elastic modulus properties of the $317 \mathrm{~L}-\mathrm{Cu}$ stainless steel

\begin{tabular}{llllll}
\hline & $\mathbf{R}_{\mathrm{p} 0.2}(\mathbf{M P a})$ & $\mathbf{R}_{\mathrm{m}}(\mathbf{M P a})$ & $\mathbf{A}(\%)$ & $\mathbf{Z}(\%)$ & $\mathbf{E ~ ( G P a )}$ \\
\hline 3I7L SS & $\geq 170$ & $\geq 485$ & $\geq 40$ & $\geq 50$ & $200 \pm I$ \\
3I7L-Cu SS & $325 \pm I$ & $632 \pm 2$ & $44 \pm 0.1$ & $47 \pm 0.5$ & $194 \pm 1$ \\
\hline
\end{tabular}

Notes: Tensile and elastic properties of 3 I 7L SS and 317L-Cu SS. Tensile properties such as yield $\left(R_{p 0.2}\right)$, tensile $\left(R_{m}\right)$ strength, elongation $(A)$, and reduction of area $(Z)$ of $317 \mathrm{~L}-\mathrm{Cu}$ SS meet the ISO 5832-I standard for $316 \mathrm{~L}$ as a surgical implant material. The elastic moduli $(\mathrm{E})$ of $317 \mathrm{~L}-\mathrm{Cu}$ SS and 317L SS are not significantly different. 


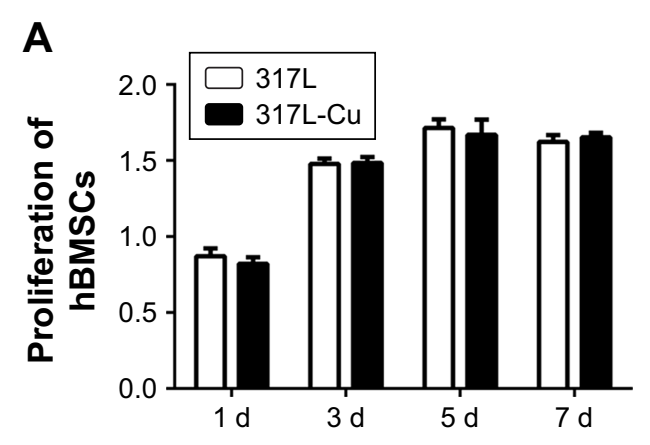

B

C

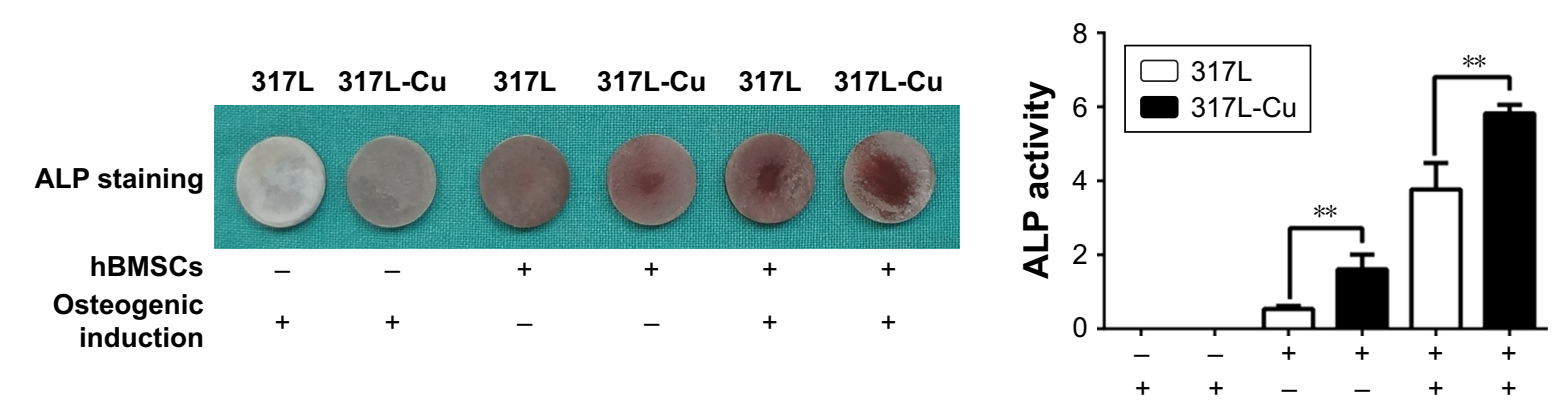

D
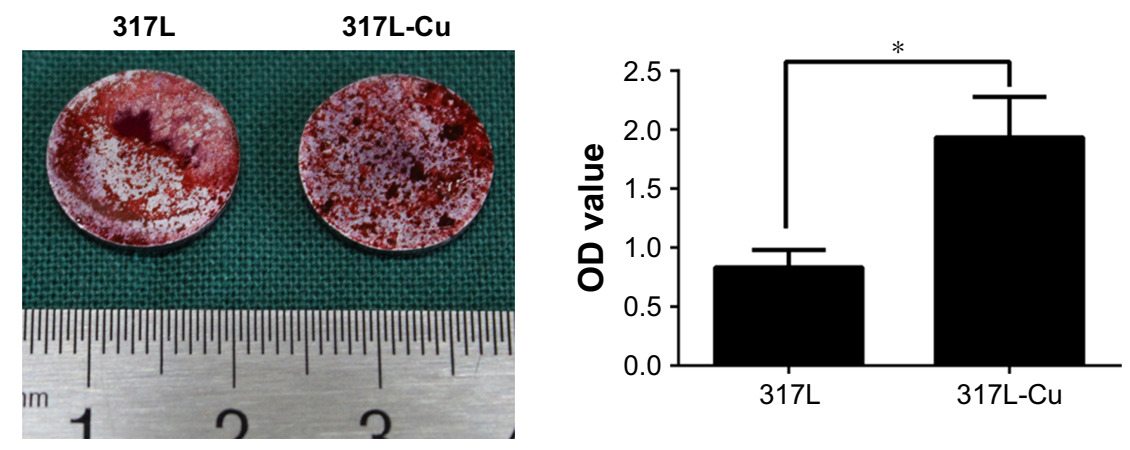

E
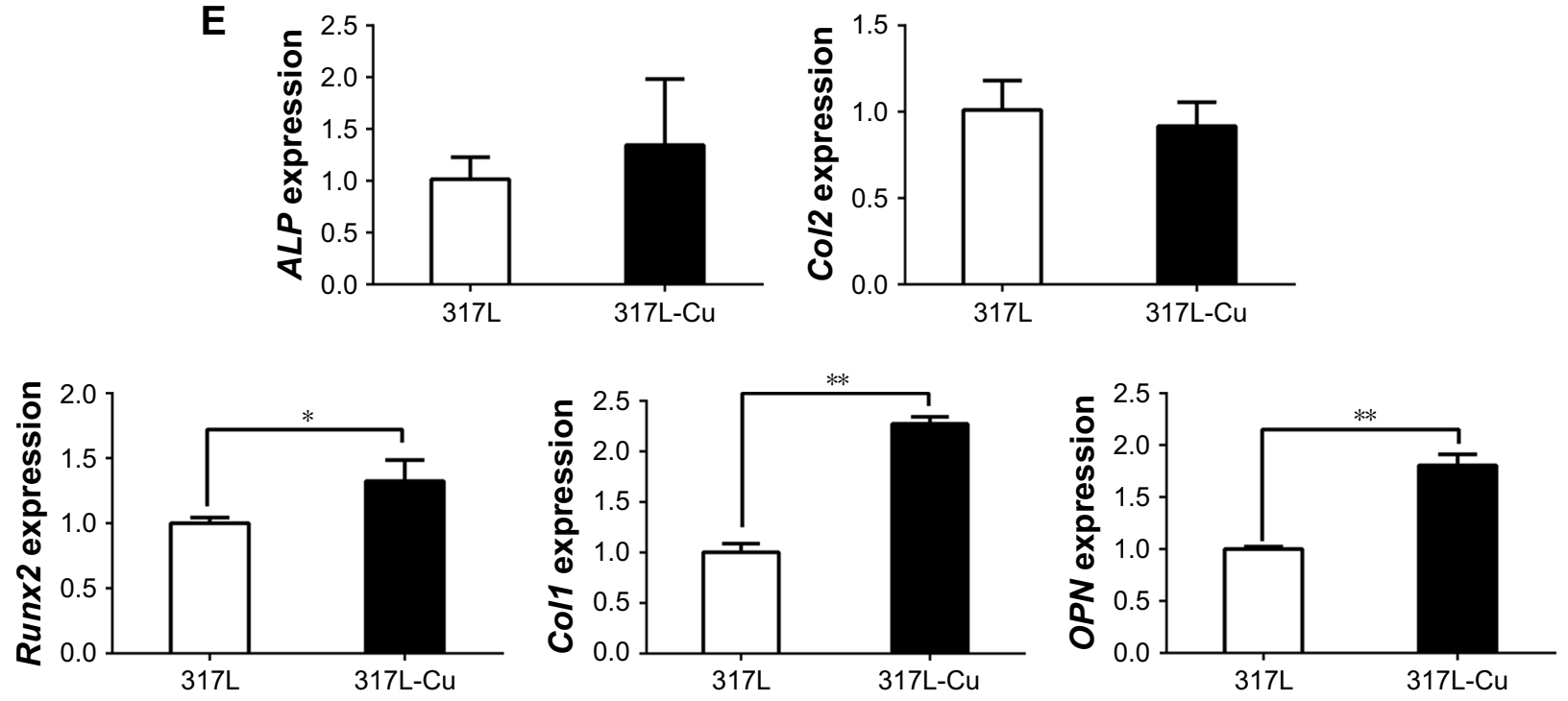

Figure I 317L-Cu stainless steel significantly promoted the osteogenic differentiation of hBMSCs without influencing their proliferation.

Notes: (A) Proliferation speed of hBMSCs on the surface of 317L-Cu SS (no significant difference compared to that of the control group). (B) hBMSCs have higher expression and activity of ALP in the 3I7L-Cu SS group, although they were not in direct contact with the material. (C and D) Better osteogenic differentiation ability, as assessed by ALP staining and alizarin red staining, is observed for hBMSCs inoculated on the surface of 3I7L-Cu SS. (E) Real-time polymerase chain reaction results indicate that 3I7L-Cu SS upregulated the expression of Runx2, OPN, and Collagen I (Coll) with statistical difference; it also promoted the expression of ALP but without significant statistical difference. There was no significant effect on the expression of Collagen 2 (Col2). $* P<0.05 ; * * P<0.01$.

Abbreviations: ALP, alkaline phosphatase; hBMSCs, human bone mesenchymal stem cells; OPN, osteopontin. 
increased expression of osteogenesis-related genes promoted by $\mathrm{Cu}^{2+}$ or the material interface.

\section{I 7L-Cu SS could significantly increase the LOX expression and activity of hBMSCs}

LOX is the key enzyme that catalyzes the cross-linking between collagen and elastin, which provides the framework for bone formation. $\mathrm{Cu}^{2+}$ is considered the most important accessory factor to determine the activity of LOX. Therefore, in order to clarify the influence of 317L-Cu SS on LOX, the expression and activity of this enzyme were detected after the hBMSCs were inoculated on the surface of 317L-Cu SS and 317L SS. First, ELISA was employed to measure the expression of LOX. Our results indicated that the secretion of LOX in both the 317L-Cu SS group and 317L SS group was significantly lower than that of hBMSCs in normal culture. Nevertheless, 317L-Cu SS could further reduce LOX secretion compared with 317L SS (Figure 2A). Furthermore, the determination of LOX activity revealed that it was significantly higher in the 317L-Cu SS group than in the control group when the cells were stimulated by these materials for 3 days (Figure 2B). Overall, although 317L-Cu SS resulted in reduced secretion of LOX, its activity was significantly promoted, which would be beneficial for the cross-linking of collagen and elastin.

\section{I7L-Cu SS was more conducive to fracture healing}

Intramedullary nails were first produced for fracture fixation (Figure 3A). SEM view of 317L-Cu SS nails showed that nano-Cu particles relatively evenly distributed in the 317L SS substrate (Figure 3B). In the X-ray images, the fracture line was visible in both groups at 3 weeks, around which a small amount of high-density bone was formed. After 6 weeks, there were obvious calluses around the ends of the fracture in both groups. However, larger calluses with cross-fracture connection were formed in the 317-Cu SS group than those in the $317 \mathrm{SS}$ group. In addition, the fracture lines were visible at this time in the 2 groups; however, the bluntness of the fracture line was greater in the $317 \mathrm{~L}-\mathrm{Cu}$ SS group than that in the 317L SS group. At 9 weeks, calluses surrounding the ends of the fractured femurs were significantly calcified into the woven bone in the $317 \mathrm{~L}-\mathrm{Cu}$ SS group, showing a further increase in the local bone density at the ends of the segments. Moreover, the fracture line almost disappeared in the 317L-Cu SS group, simultaneously, and callus remodeling was significantly better than that of the control group (Figure 3C). Further, to closely observe the healing process of the fracture, micro-CT was employed. The results showed that the callus in the $317 \mathrm{~L}-\mathrm{Cu}$ SS group had a larger volume with more bone mass than that in the $317 \mathrm{~L}$ SS group in the callus formation stage ( 3 and 6 weeks) (Figure 3D). Calluses in both groups were significantly remodeled at 9 weeks after surgery. Nevertheless, compared with the $317 \mathrm{~L}$ SS group in which the fracture line was not fully filled, the fracture line of the 317L-Cu SS group was completely filled by the bone components and its callus remodeling, accompanied by significant reduction in spongy bone, shaping of the cortical bone, and gradual increase in density (Figure 3B and E). A 3D histomorphometry of the callus tissue was carried out by micro-CT scanning, and the results are as follows: 1) callus BMD in the 317L-Cu SS group was higher than that in the control group at 3,6, and 9 weeks, with a significant difference observed between the 2 groups at 6 weeks; 2) significantly higher BV/TV was measured for the $317 \mathrm{~L}-\mathrm{Cu}$
A

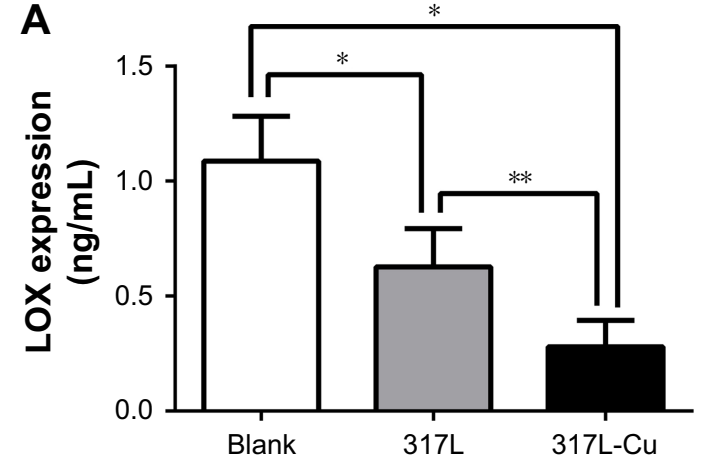

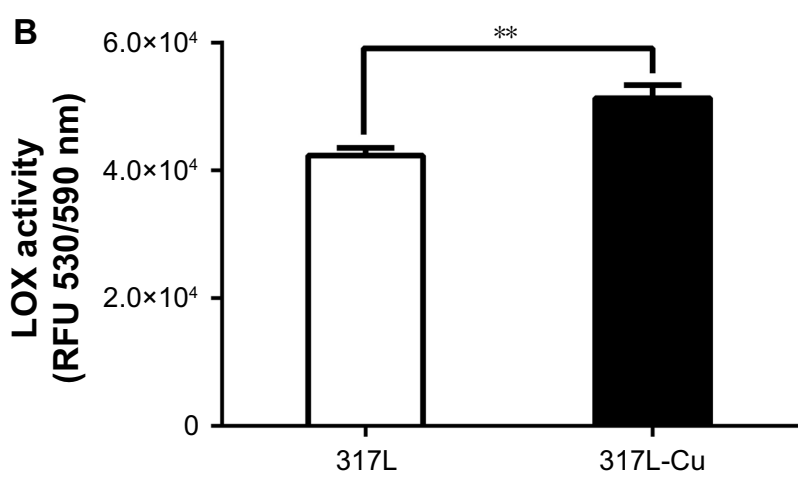

Figure 2 3I7L-Cu stainless steel significantly enhanced the activity of LOX in hBMSCs.

Notes: (A) ELISA results show that LOX secretion in both the 3I7L-Cu SS group and 3I7L SS group significantly decreased compared with that in the blank group; however, the degree of reduction of the 3I7L-Cu SS group is higher than that of the 3I7L SS group. (B) LOX activity detection indicates that 3I7L-Cu SS promotes the activity. $* P<0.05 ; * * P<0.01$.

Abbreviations: ELISA, enzyme-linked immunosorbent assay; hBMSCs, human bone mesenchymal stem cells; LOX, lysyl oxidase; RFU, relative fluorescence units. 
A

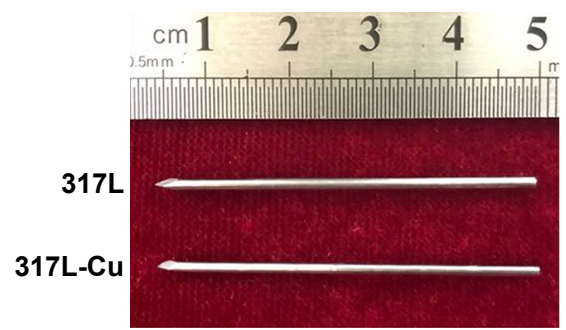

B

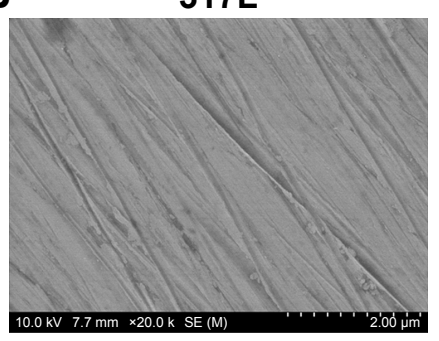

317L-Cu

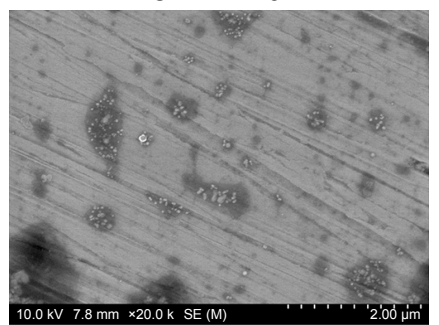

C

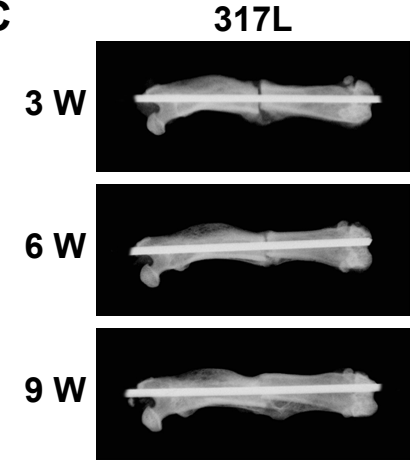

317L-Cu
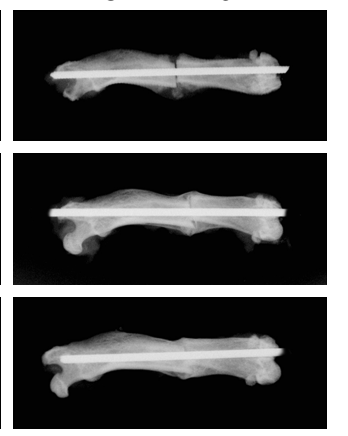

D
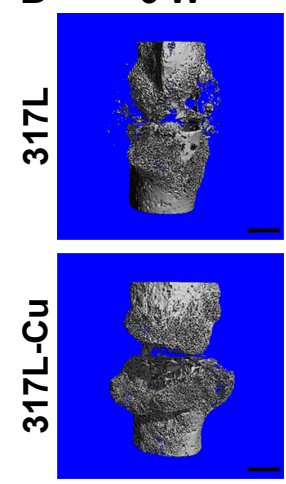

$6 \mathrm{~W}$
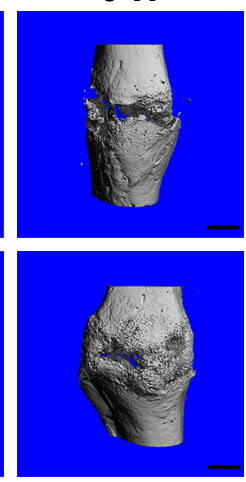

$9 \mathrm{~W}$
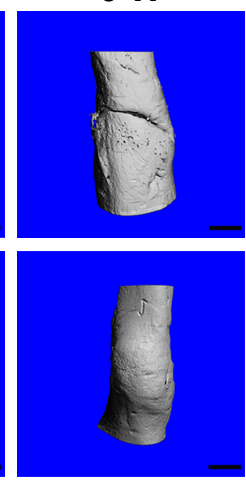

E

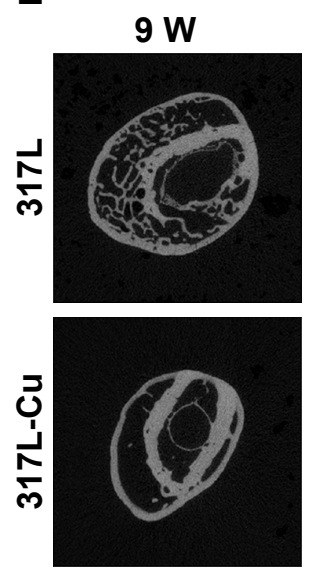

F

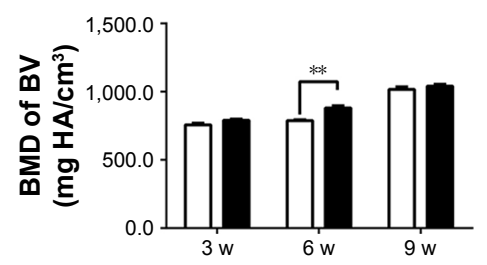

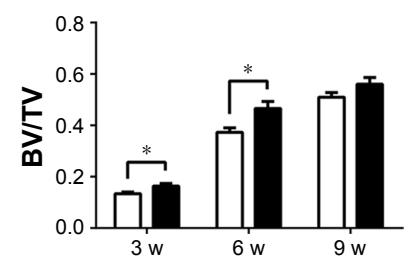
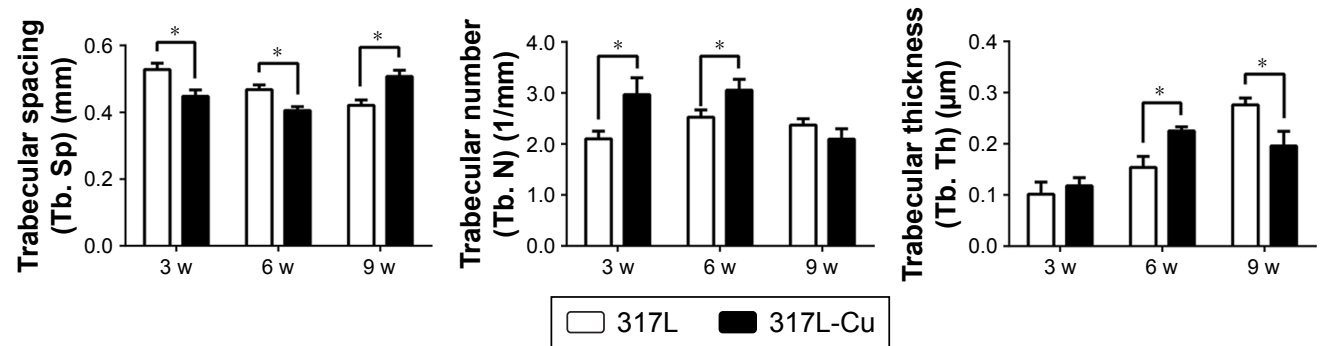

Figure 3 Radiographic assessment shows that 3I7L-Cu stainless steel is more conducive to fracture healing.

Notes: (A) General view of the intramedullary nails made by 3I7L SS and 3I7L-Cu SS. (B) SEM view of two types of intramedullary nails. Nano-copper particles evenly distributed in the 317L stainless steel substrate. (C) In X-ray, the fracture line is clearly observed in both the groups at 3 weeks after operation. At 6 weeks, calluses are formed around the ends of the fracture in both the groups, and the fracture line is largely blurred in the 3I7L-Cu SS group compared with the 3I7L SS group. At 9 weeks, the local density of the segment ends further increased in the 3I7L-Cu SS group in which the fracture line disappeared and callus remodeling significantly improved compared with the control group. ( $\mathbf{D}$ and $\mathbf{E}$ ) Micro-CT results show that the callus in the 3I7L-Cu SS group has a larger volume and more bone mass compared with those of the 3 I 7L SS group in the callus formation stage ( 3 and 6 weeks). Scale bars in B: I mm. Moreover, callus remodeling is superior to that of the control group at 9 weeks after surgery. (F) Callus BMD in the 3I7L-Cu SS group is higher than that of the control group at 3, 6, and 9 weeks, in which a significant difference between the two groups could be observed at 6 weeks. A significantly high BV/TV was measured for the 317L-Cu SS group at 3 and 6 weeks; however, the differences narrowed until they became statistically insignificant at 9 weeks. The Tb. N in 3I7L-Cu SS is greater than that of $317 \mathrm{~L} \mathrm{SS}$ at 3 and 6 weeks. Nevertheless, at 9 weeks, the Tb. N in both the groups decreased with callus remodeling but without significant differences. The Tb. Th in the 3I7L-Cu SS group significantly exceeded that of the 3I $7 \mathrm{~L} \mathrm{SS} \mathrm{group} \mathrm{at} 6$ weeks; however, an inverted effect is observed at 9 weeks in the 3 I LL-Cu SS group. The callus of 3 I 7L-Cu SS has a small Tb. Sp at 3 and 6 weeks, which significantly increased for 3 I $7 \mathrm{~L}-\mathrm{Cu}$ SS at 9 weeks. $* P<0.05$; $* * P<0.0$ I.

Abbreviations: BMD, bone mineral density; BV/TV, bone volume/tissue volume; CT, computed tomography; SEM, scanning electron microscope; Tb. N, trabecular number; Tb. Sp, trabecular spacing; Tb. Th, trabecular thickness; W, weeks.

SS group at 3 and 6 weeks, with the difference between the 2 groups narrowing due to continuous callus remodeling until 9 weeks at which time it became statistically insignificant; 3) the $\mathrm{Tb} . \mathrm{N}$ of the callus in the $317 \mathrm{~L}-\mathrm{Cu} \mathrm{SS}$ group was greater than that in the $317 \mathrm{~L}$ SS group at 3 and 6 weeks, which decreased at 9 weeks in both the groups with callus remodeling, especially in the $317 \mathrm{~L}-\mathrm{Cu}$ SS group, but without significant differences between the 2 groups; 4 ) the $\mathrm{Tb}$. Th of the callus in the $317 \mathrm{~L}-\mathrm{Cu}$ SS group at 6 weeks significantly exceeded that in the $317 \mathrm{~L}$ SS group, with an interesting 
inverted effect observed at 9 weeks, which possibly resulted from the higher callus remodeling efficiency in the $317 \mathrm{~L}-\mathrm{Cu}$ SS group compared with that of the 317L SS group; and 5 ) the callus of $317 \mathrm{~L}-\mathrm{Cu}$ SS had a small Tb. Sp at 3 and 6 weeks, which might be related to the significant increase in $\mathrm{Tb}$. $\mathrm{N}$ and $\mathrm{Tb}$. Th caused by the osteogenic promotion effect of $\mathrm{Cu}^{2+}$. However, Tb. Sp was significantly increased compared with that in the control group due to the rapid reconstruction of trabecular bone at 9 weeks after surgery (Figure 3F). Overall, it could be inferred that 317L-Cu SS not only promoted osteogenesis in the callus but also upregulated the callus remodeling efficiency, leading to an early mechanical stability recovery of the fractured femur.

\section{I7L-Cu SS could accelerate the callus evolution by promoting its collagen formation, osteogenesis and remodeling}

At 3 weeks, histological evaluation after Masson staining showed that the calluses of the 2 groups were mainly composed of fiber components, and the amount of fibrous tissue of the $317 \mathrm{~L}-\mathrm{Cu}$ SS group was significantly higher than that of the 317L SS group. However, cartilage and bone-like components were also observed at both the ends of the calluses in the 2 groups, with the $317 \mathrm{~L}-\mathrm{Cu}$ SS group exhibiting a higher amount (Figure 4A and B). At the 6th week, extensive new bone formation was observed in both groups, which enabled the broken ends of the femurs to be connected in the calluses (Figure 4A). Moreover, the safranin $\mathrm{O} /$ fast green staining image shown in Figure 4C reveals a higher amount of cartilage component in the $317 \mathrm{~L}-\mathrm{Cu}$ SS group than that in the 317L SS group at either 3 weeks or 6 weeks after surgery. The Van Gieson staining images for each time point $(3,6$, and 9 weeks) for both groups are shown in Figure 4D. A large new cancellous bone formation area was observed in the callus of the 317L-Cu SS group at 6 weeks, which became smaller at 9 weeks, compared with the callus of the $317 \mathrm{~L}$ SS group. However, a large amount of thick cortical bone was formed at 9 weeks in the 317L-Cu SS group. Calcein/ alizarin red double labeling also indicated accelerated new bone formation in the 317L-Cu SS group at 3 and 6 weeks compared with that in the $317 \mathrm{~L}$ SS group. Besides, with the commencement of callus remodeling, the bone formation rate in both groups began to decrease, especially in the $317 \mathrm{~L}-\mathrm{Cu}$ SS group, although there was no statistically significant difference between the 2 groups (Figure 4E). Collectively, these results further confirm that the new material promotes bone formation in the callus and subsequently accelerates callus remodeling.

\section{I 7L-Cu SS could significantly increase $\mathrm{Cu}^{2+}$ content in the callus and further enhance the expression of Runx 2 in the fracture local site}

To preliminarily explore the possible reasons for the aforementioned effects of $317 \mathrm{~L}-\mathrm{Cu}$ SS, some samples of either group were ground into homogenized tissue fluid for $\mathrm{Cu}^{2+}$ detection. Our results showed that there was a significant increase in $\mathrm{Cu}^{2+}$ content in the calluses of the $317 \mathrm{~L}-\mathrm{Cu}$ SS group compared with that of the calluses in the control group at 3, 6, and 9 weeks, which suggests that the increased $\mathrm{Cu}^{2+}$ content might be the basis for $317 \mathrm{~L}-\mathrm{Cu}$ SS to promote fracture healing and remodeling (Figure 5A). To confirm this speculation, we extracted the callus protein to examine the expression of Runx2. As expected, Runx2 expression in the $317 \mathrm{~L}-\mathrm{Cu}$ SS group was significantly higher than that in the $317 \mathrm{~L} \mathrm{SS}$ group at 3 and 6 weeks (Figure 5B). In addition, serum $\mathrm{Cu}^{2+}$ in both the groups was determined, showing that 317L-Cu SS had no effect on the serum $\mathrm{Cu}^{2+}$ concentration. Therefore, 317L-Cu SS used for fracture fixation should not have systemic toxicity (Figure 5C).

\section{I $7 \mathrm{~L}-\mathrm{Cu}$ SS was conducive to the recovery of mechanical properties of fractured femur}

The 317L-Cu SS group exhibited superior biomechanical properties compared to the $317 \mathrm{~L} \mathrm{SS}$ group in the 3 -point bending test. For the control group, the maximum load tolerated by the entire bone segment (including the osteotomy gap), measured at 3,6, and 9 weeks after the index procedure, was $22.94 \pm 1.43 \mathrm{~N}, 65.23 \pm 8.57 \mathrm{~N}$, and $91.1 \pm 3.97 \mathrm{~N}$, respectively. In comparison, the maximum loads tolerated by $317 \mathrm{~L}-\mathrm{Cu}$ SS were significantly higher for the same time points; that is, $25.83 \pm 2.33 \mathrm{~N}, 80.04 \pm 7.86$ $\mathrm{N}(P<0.05)$, and $109.54 \pm 7.36 \mathrm{~N}(P<0.05)$ at 3,6 , and 9 weeks, respectively. However, both groups demonstrated improved bending strength with fracture healing and gradual callus remodeling (Figure 6).

\section{Discussion}

Modern orthopedic fracture fixation implants are typically manufactured using 1 of the 3 available metals: grade $316 \mathrm{~L}$ or $317 \mathrm{~L} \mathrm{SS}$, commercially pure titanium, or titanium alloy (typically type Ti-6Al-4V, although other alloys are available). ${ }^{31}$ Stiffness and fatigue strength are generally considered as most important properties of metals for fracture fixation, and all the 3 aforementioned materials meet the basic clinical requirements in this regard. However, in the 


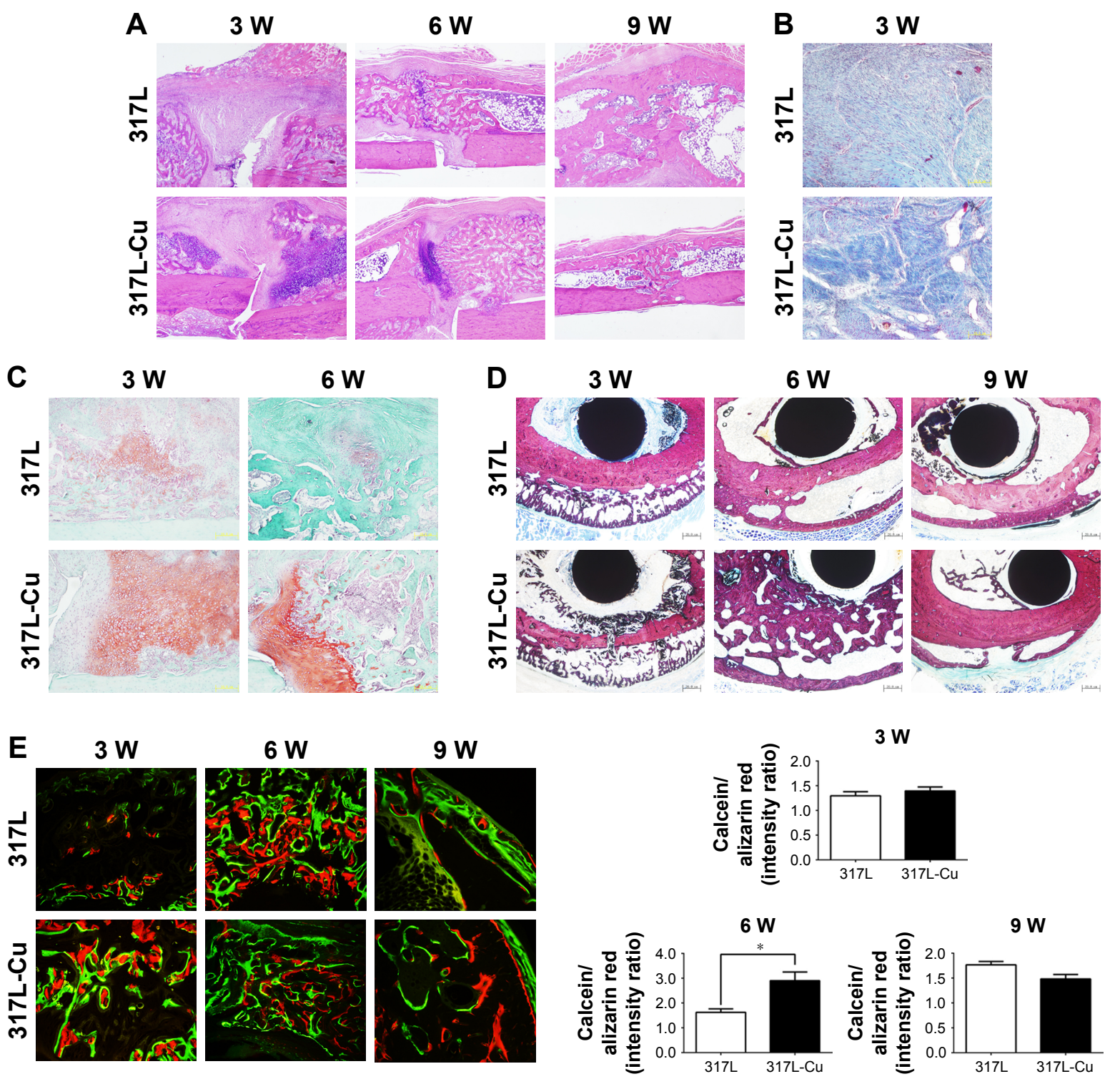

Figure 4 3I7L-Cu stainless steel accelerated callus evolution by promoting collagen formation, osteogenesis, and remodeling, as observed through histological assessment. Notes: (A) H\&E staining displays the general evolution of calluses. At 3 weeks, the calluses of the two groups are mainly composed of fiber components. At 6 weeks, new bone formed with greater volume in the 3I7L-Cu SS group. By the 9th week, the remodeling of the callus of the 3I7L-Cu SS group was better. ( $\times 40)$. (B) The amount of fibrous tissue indicated in blue in the 317L-Cu SS group is significantly higher than that in the 3I7L SS group, as seen by Masson staining $(\times 100)$. (C) Safranin O/fast green staining shows that the 3I7L-Cu SS group has a larger amount of cartilage components (indicated in red) than 3I7L SS group does at both 3 and 6 weeks after surgery $(\times 100)$. (D) Van Gieson staining shows a large new cancellous bone formation area, displayed in red, in the 317L-Cu SS group at 6 weeks, which decreased at 9 weeks, compared with the control group. However, a large amount of thick cortical bone formed at 9 weeks in the 3I7L-Cu SS group $(\times 40)$. (E) Calcein/Alizarin red double labeling indicates accelerated new bone formation in the 317L-Cu SS group at 3 and 6 weeks compared to that in the 317L SS group. At 9 weeks, the callus remodeling of the 317L-Cu SS group was better than that of the control group $(\times 200)$. $* P<0.05$.

Abbreviation: W, weeks.

event of delayed union or nonunion, these materials will be insufficient because they cannot promote the formation and remodeling of callus. On the other hand, several issues need to be addressed to develop a completely new type of fracture fixation material; for example, it needs to be ensured that the mechanical properties meet the industrial criteria, biocompatibility is in the allowable range, and material cost is controllable, which might involve a protracted process. Therefore, property modification of the existing clinical metal materials will not only be the most cost-effective, but also the most beneficial for clinical transformation. The $317 \mathrm{~L}-\mathrm{Cu}$ SS was developed based on this concept. We confirmed that the new material has good biocompatibility without changes in serum $\mathrm{Cu}^{2+}$ concentration and liver damage ${ }^{27}$ and almost does not lead to additional production cost. Importantly, our results in this study further prove that the new material preserves the mechanical properties of stainless steel without significant difference. Consequently, we expect that $317 \mathrm{~L}-\mathrm{Cu}$ 

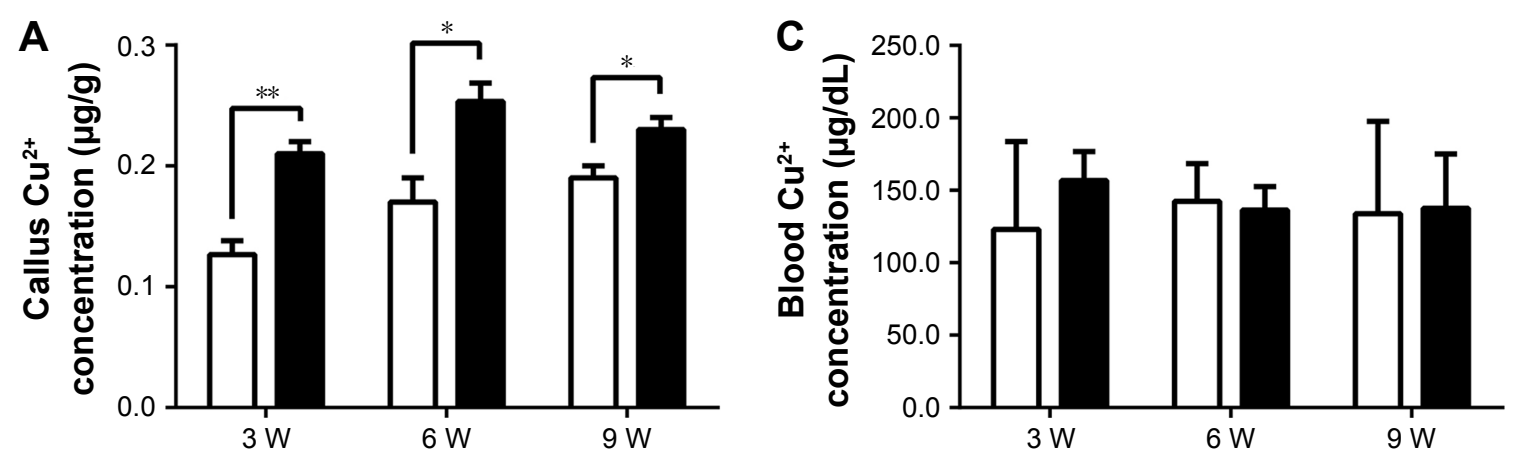

$317 \mathrm{~L}$

$317 \mathrm{~L}-\mathrm{Cu}$

B
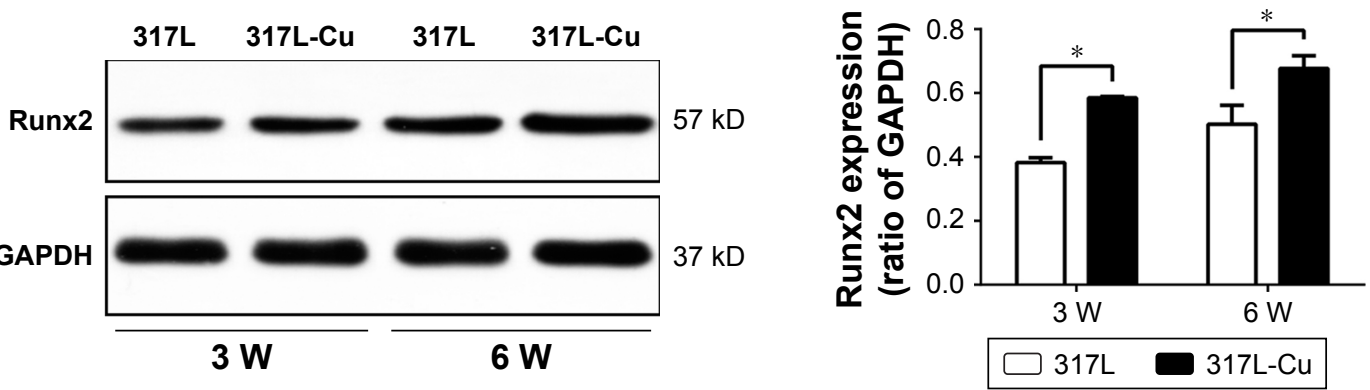

Figure 5 3I $7 \mathrm{~L}-\mathrm{Cu}$ stainless steel significantly increased the $\mathrm{Cu}^{2+}$ content in the callus and further enhanced the expression of Runx2 in the fracture local site.

Notes: (A) Significant increase in $\mathrm{Cu}^{2+}$ content in the calluses of the 317L-Cu SS group compared with that of the control group at 3, 6, and 9 weeks. (B) Runx2 expression in the $317 \mathrm{~L}-\mathrm{Cu}$ SS group is significantly higher than that in the $317 \mathrm{~L} \mathrm{SS}$ group at 3 and 6 weeks. (C) $317 \mathrm{~L}-\mathrm{Cu}$ SS could not increase the concentration of serum Cu ${ }^{2+}$. $* P<0.05$; $* * \mathrm{P}<0.01$

Abbreviations: GAPDH, glyceraldehyde-3-phosphate dehydrogenase; W, weeks.

SS can be directly used in clinical practice, although a rigorous preclinical trial is needed.

Recently, many laboratory studies have shown that increased $\mathrm{Cu}^{2+}$ could induce the differentiation of mesenchymal stem cells and osteoblastic cells. ${ }^{32,33} \mathrm{Wu}$ et al ${ }^{34}$ incorporated $\mathrm{Cu}^{2+}$ into a mesoporous bioactive glass (MBG) scaffold, and demonstrated that the Cu-containing MBG scaffold

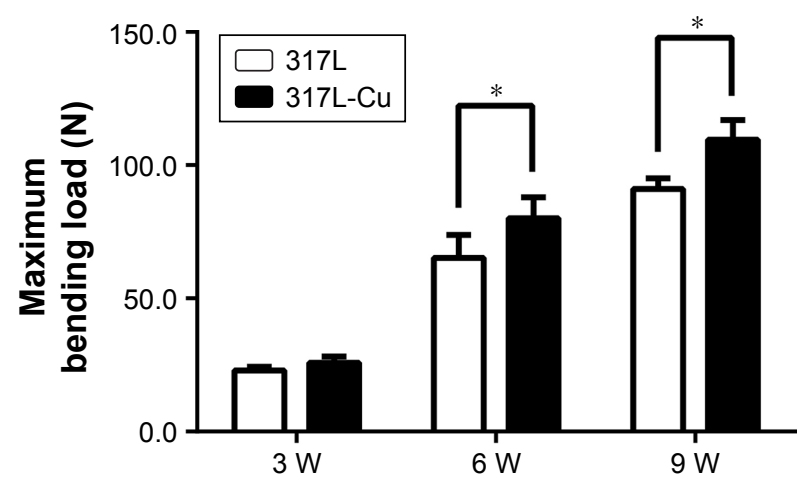

Figure 6 3I7L-Cu stainless steel is conducive to the recovery of mechanical properties of the fractured femur.

Notes: The maximum loads tolerated by the entire bone segment (including the osteotomy gap) in the 3 I L L SS group, measured at 3,6, and 9 weeks (W) after the index procedure, are $22.94 \pm 1.43 \mathrm{~N}, 65.23 \pm 8.57 \mathrm{~N}$, and $91.1 \pm 3.97 \mathrm{~N}$, respectively. In comparison, the maximum loads tolerated by 317L-Cu SS are significantly higher at the same time points; that is, $25.83 \pm 2.33 \mathrm{~N}, 80.04 \pm 7.86 \mathrm{~N}(P<0.05)$, and $109.54 \pm 7.36 \mathrm{~N}(P<0.05)$ at 3,6 , and 9 weeks, respectively. $* P<0.05$. possessed obvious ability of osteostimulation due to the release of $\mathrm{Cu}^{2+}$ that played a central role to stimulate the ALP activity and the osteogenic gene expression. And recently, Prinz et $\mathrm{al}^{35}$ also found that a Cu-coated implant stimulated bone formation by releasing $\mathrm{Cu}$ ions and concluded that the use of implants with a defined load of $\mathrm{Cu}$ ions enables both prevention of bacterial infection and the stimulation of regenerative processes. Therefore, in order to realize the application of $317 \mathrm{~L}-\mathrm{Cu}$ SS in the treatment of orthopedic diseases, its effect on fracture healing was studied in this article. As is known, fracture is the most common disease in orthopedics, and callus formation and remodeling are the most important factors for fracture healing. Generally, callus osteogenesis can occur in 2 ways: endochondral ossification and periosteal ossification. In endochondral ossification, the new bone forms from the transformation of fibrous callus to cartilage callus and the subsequent hyperplasia and calcification of cartilage callus. However, in periosteal ossification, the new bone is formed by the proliferation and differentiation of osteogenesis-related cells in the periosteum, which is also the main route to generating a hard callus. ${ }^{36,37}$ It is known that hard callus is the key structure to close the fracture gap and connect both ends, finally leading to preliminary stability and recovery of biomechanical functions 
for the fractured bone. ${ }^{38}$ Subsequently, the callus gradually remodels with time. The arrangement of trabecular bone (from loose to tight) and the formation of bony connections are the main steps in this process. At this stage, the broken bone has sufficient mechanical stability and the fracture has undergone basic healing. Therefore, shortening the evolution process of callus and improving callus quality will determine the fracture healing speed and its prognosis. Our results proved that $317 \mathrm{~L}-\mathrm{Cu}$ SS possesses this ability. 317L-Cu SS could significantly promote the osteogenic differentiation of hBMSCs, a very important factor for fracture healing, ${ }^{39}$ which would migrate into the callus and subsequently undergo osteogenic differentiation to facilitate the fracture healing process. ${ }^{40}$ Moreover, such osteogenic effects of 317L-Cu SS might derive from its unique interface and the released $\mathrm{Cu}^{2+}$, further increasing the expression of the key osteogenesis-related factors, especially Runx $2 .{ }^{41,42}$ When the material is placed in the medullary cavity of the fractured bone, the interface should not affect the osteogenesis of the callus due to the lack of direct contact. Interestingly, it was shown that the $\mathrm{Cu}^{2+}$ concentration of the callus in the 317L-Cu SS group significantly increased compared with that of the callus in the control group, implying that its role in promoting bone formation was primarily due to the released $\mathrm{Cu}^{2+}$ after implantation in the body. Regrettably, we were unable to determine whether the elevated $\mathrm{Cu}^{2+}$ mainly occurred intracellularly or extracellularly because there are no appropriate technical means for the detection of $\mathrm{Cu}^{2+}$ distribution in vivo.

On the other hand, $\mathrm{Cu}^{2+}$ as an important prothetic group can upregulate the activity of LOX, which then oxidizes the specific amino acid residues in collagen and elastin and promotes covalent bond formation between the 2 proteins. ${ }^{43,44}$ Fibrous tissue is essential in the process of fracture healing. The fibrous callus is the main structure responsible for the early connection of the fracture ends. In the process of fibrous callus formation and transformation, LOX may catalyze lysine and hydroxyl lysine side chains to form aldehydes, leading to the cross-linking of fiber collagens. ${ }^{45}$ The crosslinking then facilitates callus osteogenesis and provides the necessary framework for bone mineralization. ${ }^{46}$ In this work, we proved through the in vitro experiment that $317 \mathrm{~L}-\mathrm{Cu}$ SS has significant effect in enhancing the activity of LOX in hBMSCs. It is possibly because of this that more of the woven fibrous tissue was observed through Masson staining in the early stage of callus formation in the $317 \mathrm{~L}-\mathrm{Cu} \mathrm{SS}$ group. Therefore, it was inferred that the fibrous callus could form more quickly and a better "foundation" was provided for the subsequent callus osteogenesis.

\section{The top of the form}

Faster callus remodeling was also observed under the influence of $317 \mathrm{~L}-\mathrm{Cu} \mathrm{SS}$ in our research through radiographic and histological investigations. As is known, osteoclasts are necessary for the remodeling of callus into mature bone. ${ }^{47,48}$ Although the effect of $317 \mathrm{~L}-\mathrm{Cu}$ SS on the functions of osteoclasts is not clear, $\mathrm{Cu}^{2+}$ seems to have some effect on osteoclasts, which still needs further study. $\mathrm{Li}$ and $\mathrm{Yu}$ found that extracellular $\mathrm{Cu}^{2+}$ could inhibit osteoclastic resorption on dental slices ${ }^{49}$ However, in the investigation of the mechanism of colorectal cancer colonization in bone, LOX was found acting in concert to promote receptor activator of nuclear factor kappa-B ligand-dependent osteoclast differentiation, indicating that $\mathrm{Cu}^{2+}$ could indirectly affect osteoclasts. ${ }^{50}$ Therefore, further research is needed to verify whether the accelerated callus remodeling effectuated by $317 \mathrm{~L}-\mathrm{Cu} \mathrm{SS}$ is associated with the change in the function of osteoclasts. What is more, this phenomenon may also have eventuated from the promotion of formation of fibrous callus and callus osteogenesis by $317 \mathrm{~L}-\mathrm{Cu}$ SS, followed by an early access to callus remodeling. Consequently, 317L-Cu SS resulted in faster completion of callus remodeling and fracture healing.

\section{Conclusion}

317L-Cu SS promoted fracture healing via 3 mechanisms: 1) $\mathrm{Cu}^{2+}$ released by $317 \mathrm{~L}-\mathrm{Cu} \mathrm{SS}$ significantly upregulated the LOX activity to further promote the cross-linking between collagen and elastin, thus establishing the support structure for callus ossification and mineralization; 2) 317L-Cu SS induced the osteogenesis of hBMSCs, providing the "prime power" for callus ossification; and 3) 317L-Cu SS accelerated the callus evolution process, though the specific mechanisms require further exploration. Nonetheless, our present and previous results fully demonstrate that $317 \mathrm{~L}-\mathrm{Cu}$ SS not only possesses good biocompatibility but also considerable potential for the treatment of orthopedic diseases.

\section{Acknowledgment}

This work was supported by grants from the National Key Research and Development Program of China (No 2016YFC1100600), the National Natural Science Foundation of China (No 81071472, No 81371960, and No 51631009), the Key National Basic Research Program of China (2012CB619101), the Technology Support Project of the Science and Technology Commission of Shanghai (No 13441901302, No 14441901000 and No 15411951200), the New Cutting-Edge Technology Project of ShenKang Hospital Development Center of Shanghai (SHDC12014124), the Shanghai Jiao Tong University Cross Research Fund of 
Medical Engineering (YG2013MS57), the Multicenter Clinical Research Project of Shanghai Jiao Tong University School of Medicine (DLY201506), and Doctoral Innovation Fund Projects from Shanghai Jiao Tong University School of Medicine (BXJ201429).

\section{Disclosure}

The authors report no conflicts of interest in this work.

\section{References}

1. Ghiasi MS, Chen J, Vaziri A, Rodriguez EK, Nazarian A. Bone fracture healing in mechanobiological modeling: a review of principles and methods. Bone Rep. 2017;6:87-100.

2. Cottrell J, O'Connor JP. Effect of non-steroidal anti-inflammatory drugs on bone healing. Pharmaceuticals (Basel). 2010;3(5):1668-1693.

3. Pivonka P, Dunstan CR. Role of mathematical modeling in bone fracture healing. Bonekey Rep. 2012;1:221.

4. Bonafede M, Espindle D, Bower AG. The direct and indirect costs of long bone fractures in a working age US population. J Med Econ. 2013; 16(1):169-178.

5. Einhorn TA. Enhancement of fracture-healing. J Bone Joint Surg Am. 1995;77(6):940-956.

6. Einhorn TA, Gerstenfeld LC. Fracture healing: mechanisms and interventions. Nat Rev Rheumatol. 2015;11(1):45-54.

7. Darouiche RO. Treatment of infections associated with surgical implants. N Engl J Med. 2004;350(14):1422-1429.

8. Tay WH, de Steiger R, Richardson M, Gruen R, Balogh ZJ. Health outcomes of delayed union and nonunion of femoral and tibial shaft fractures. Injury. 2014;45(10):1653-1658.

9. Hak DJ, Fitzpatrick D, Bishop JA, et al. Delayed union and nonunions: epidemiology, clinical issues, and financial aspects. Injury. 2014; 45(Suppl 2):S3-S7.

10. Metsemakers WJ, Moriarty TF, Nijs S, Pape HC, Richards RG. Influence of implant properties and local delivery systems on the outcome in operative fracture care. Injury. 2016;47(3):595-604.

11. Zhao Y, Cao H, Qin H, et al. Balancing the osteogenic and antibacterial properties of titanium by codoping of $\mathrm{Mg}$ and $\mathrm{Ag}$ : an in Vitro and in Vivo Study. ACS Appl Mater Interfaces. 2015;7(32):17826-17836.

12. Kumar V, Prakash J, Singh JP, et al. Role of silver doping on the defects related photoluminescence and antibacterial behaviour of zinc oxide nanoparticles. Colloids Surf B Biointerfaces. 2017;159:191-199.

13. Lai M, Jin Z, Su Z. Surface modification of $\mathrm{TiO} 2$ nanotubes with osteogenic growth peptide to enhance osteoblast differentiation. Mater Sci Eng C Mater Biol Appl. 2017;73:490-497.

14. Bíbr B, Deyl Z, Lener J, Kucera J, Simková M. The mechanism of action of molybdenum and tungsten upon collagen structures in vivo. Physiol Bohemoslov. 1987;36(5):417-424.

15. McGraw KJ. Melanins, metals, and mate quality. Oikos. 2003;102(2): 402-406.

16. Uauy R, Olivares M, Gonzalez M. Essentiality of copper in humans. Am J Clin Nutr. 1998;67(5 Suppl):952S-959S.

17. Gupta A, Lutsenko S. Human copper transporters: mechanism, role in human diseases and therapeutic potential. Future Med Chem. 2009; 1(6):1125-1142.

18. Dong Y, Li X, Bell T, Sammons R, Dong H. Surface microstructure and antibacterial property of an active-screen plasma alloyed austenitic stainless steel surface with $\mathrm{Cu}$ and N. Biomed Mater. 2010;5(5):054105.

19. Wu Y, Han G, Gong Y, et al. Antibacterial property and mechanism of copper alginate fiber. Adv Mater Res. 2011;152-153:1351-1355.

20. Wu C, Zhou Y, Xu M, et al. Copper-containing mesoporous bioactive glass scaffolds with multifunctional properties of angiogenesis capacity, osteostimulation and antibacterial activity. Biomaterials. 2013; 34(2):422-433.
21. Barceló-Oliver M, García-Raso A, Terrón A, et al. Synthesis and mass spectroscopy kinetics of a novel ternary copper(II) complex with cytotoxic activity against cancer cells. J Inorg Biochem. 2007;101(4): 649-659.

22. Hubacher D, Lara-Ricalde R, Taylor DJ, Guerra-Infante F, GuzmánRodríguez R. Use of copper intrauterine devices and the risk of tubal infertility among nulligravid women. $N$ Engl J Med. 2001;345(8): 561-567.

23. Hoene A, Prinz C, Walschus U, et al. In vivo evaluation of copper release and acute local tissue reactions after implantation of coppercoated titanium implants in rats. Biomed Mater. 2013;8(3):035009.

24. Hoene A, Patrzyk M, Walschus U, et al. In vivo examination of the local inflammatory response after implantation of Ti6Al4V samples with a combined low-temperature plasma treatment using pulsed magnetron sputtering of copper and plasma-polymerized ethylenediamine. J Mater Sci Mater Med. 2013;24(3):761-771.

25. Bartsch I, Willbold E, Yarmolenko S, Witte F. In vivo fluorescence imaging of apoptosis during foreign body response. Biomaterials. 2012;33(29):6926-6932.

26. Ren L, Wong HM, Yan CH, Yeung KW, Yang K. Osteogenic ability of Cu-bearing stainless steel. J Biomed Mater Res B Appl Biomater. 2015; 103(7):1433-1444.

27. Wang L, Ren L, Tang T, Dai K, Yang K, Hao Y. A novel nano-copperbearing stainless steel with reduced $\mathrm{Cu}(2+)$ release only inducing transient foreign body reaction via affecting the activity of NF- $\kappa \mathrm{B}$ and Caspase 3. Int J Nanomedicine. 2015;10:6725-6739.

28. Sun D, Xu D, Yang C, et al. An investigation of the antibacterial ability and cytotoxicity of a novel cu-bearing $317 \mathrm{~L}$ stainless steel. Sci Rep. 2016;6:29244.

29. Pittenger MF, Mackay AM, Beck SC, et al. Multilineage potential of adult human mesenchymal stem cells. Science. 1999;284(5411):143-147.

30. Yang F, Xie Y, Li H, et al. Human bone marrow-derived stromal cells cultured with a plasma sprayed $\mathrm{CaO}-\mathrm{ZrO} 2-\mathrm{SiO} 2$ coating. J Biomed Mater Res B Appl Biomater. 2010;95(1):192-201.

31. Beltran MJ, Collinge CA, Gardner MJ. Stress modulation of fracture fixation implants. J Am Acad Orthop Surg. 2016;24(10):711-719.

32. Ewald A, Käppel C, Vorndran E, Moseke C, Gelinsky M, Gbureck U. The effect of $\mathrm{Cu}(\mathrm{II})$-loaded brushite scaffolds on growth and activity of osteoblastic cells. J Biomed Mater Res A. 2012;100(9):2392-2400.

33. Rodríguez JP, Ríos S, González M. Modulation of the proliferation and differentiation of human mesenchymal stem cells by copper. $J$ Cell Biochem. 2002;85(1):92-100.

34. Wu C, Zhou Y, Xu M, et al. Copper-containing mesoporous bioactive glass scaffolds with multifunctional properties of angiogenesis capacity, osteostimulation and antibacterial activity. Biomaterials. 2013; 34(2):422-433.

35. Prinz C, Elhensheri M, Rychly J, Neumann HG. Antimicrobial and bone-forming activity of a copper coated implant in a rabbit model. J Biomater Appl. 2017;32(2):139-149.

36. Fazzalari NL. Bone fracture and bone fracture repair. Osteoporos Int. 2011;22(6):2003-2006.

37. Neagu TP, Ţigliş M, Cocoloş I, Jecan CR. The relationship between periosteum and fracture healing. Rom J Morphol Embryol. 2016;57(4): 1215-1220.

38. Gerstenfeld LC, Alkhiary YM, Krall EA, et al. Three-dimensional reconstruction of fracture callus morphogenesis. J Histochem Cytochem. 2006;54(11):1215-1228.

39. Watson L, Elliman SJ, Coleman CM. From isolation to implantation: a concise review of mesenchymal stem cell therapy in bone fracture repair. Stem Cell Res Ther. 2014;5(2):51.

40. Taguchi K, Ogawa R, Migita M, Hanawa H, Ito H, Orimo H. The role of bone marrow-derived cells in bone fracture repair in a green fluorescent protein chimeric mouse model. Biochem Biophys Res Commun. 2005;331(1):31-36.

41. Xu J, Li Z, Hou Y, Fang W. Potential mechanisms underlying the Runx2 induced osteogenesis of bone marrow mesenchymal stem cells. Am J Transl Res. 2015;7(12):2527-2535. 
42. Vimalraj S, Arumugam B, Miranda PJ, Selvamurugan N. Runx2: Structure, function, and phosphorylation in osteoblast differentiation. Int J Biol Macromol. 2015;78:202-208.

43. Hornstra IK, Birge S, Starcher B, Bailey AJ, Mecham RP, Shapiro SD. Lysyl oxidase is required for vascular and diaphragmatic development in mice. J Biol Chem. 2003;278(16):14387-14393.

44. Liu X, Zhao Y, Pawlyk B, Damaser M, Li T. Failure of elastic fiber homeostasis leads to pelvic floor disorders. Am J Pathol. 2006;168(2): 519-528.

45. Eyre DR, Paz MA, Gallop PM. Cross-linking in collagen and elastin. Annu Rev Biochem. 1984;53:717-748.

46. Khosravi R, Sodek KL, Xu WP, et al. A novel function for lysyl oxidase in pluripotent mesenchymal cell proliferation and relevance to inflammation-associated osteopenia. PLoS One. 2014;9(6):e100669.
47. Wang T, Zhang X, Bikle DD. Osteogenic differentiation of periosteal cells during fracture healing. J Cell Physiol. 2017;232(5):913-921.

48. Schindeler A, McDonald MM, Bokko P, Little DG. Bone remodeling during fracture repair: the cellular picture. Semin Cell Dev Biol. 2008; 19(5):459-466.

49. Li BB, Yu SF. [In vitro study of the effects of copper ion on osteoclastic resorption in various dental mineralized tissues.] Zhonghua Kou Qiang Yi Xue Za Zhi. 2007;42(2):110-113. Chinese.

50. Reynaud C, Ferreras L, Di Mauro P, et al. Lysyl oxidase is a strong determinant of tumor cell colonization in bone. Cancer Res. 2017;77(2): 268-278.
International Journal of Nanomedicine

\section{Publish your work in this journal}

The International Journal of Nanomedicine is an international, peerreviewed journal focusing on the application of nanotechnology in diagnostics, therapeutics, and drug delivery systems throughout the biomedical field. This journal is indexed on PubMed Central, MedLine, CAS, SciSearch $®$, Current Contents $\AA /$ Clinical Medicine,

\section{Dovepress}

Journal Citation Reports/Science Edition, EMBase, Scopus and the Elsevier Bibliographic databases. The manuscript management system is completely online and includes a very quick and fair peer-review system, which is all easy to use. Visit http://www.dovepress.com/ testimonials.php to read real quotes from published authors.

Submit your manuscript here: http://www.dovepress.com/international-journal-of-nanomedicine-journal 